
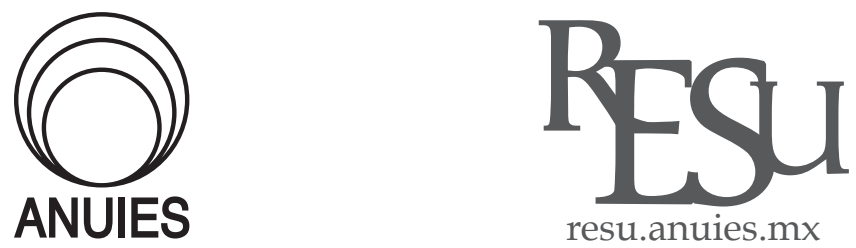

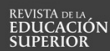

ARTíCULO

\title{
Sobre la función social del conocimiento humano mediante la vinculación y transferencia del conocimiento en América Latina*
}

\author{
The social function of knowledge through linking in Knowledge Transfer in \\ Latin America
}

\section{Claudia Rivera Hernández**}

* Agradecimientos: Agradezco a la Benemérita Universidad Autónoma de Puebla por el apoyo recibido y por la oportunidad de formar parte de esta gran comunidad académica. Este documento es parte del proyecto de investigación financiado por la Vicerrectoría de Investigación y Estudios de Posgrado de la BUAP

** Benemérita Universidad Autónoma de Puebla. Correo electrónico: claudia.rivera@correo.buap.mx

Recibido el 28 de junio del 2017; aceptado el 22 de febrero del 2019.

\section{Resumen}

A partir del análisis histórico de la vinculación y la transferencia del conocimiento en las Universidades de América Latina, este documento presenta una alternativa de vinculación basada en la Responsabildiad Social Universitaria. Para ello se presenta una serie de estrategias y propuestas que desde esta optica favorecen la función social del conocimiento producido en las Universidades públicas como agentes de cambio en la búsqueda del desarrollo equitativo en contextos locales.

Palabras clave: Educación Superior; Responsabilidad Social; Política Educativa; Vinculación y Transferencia del conocimiento. 


\begin{abstract}
This document analized the theoretical concept of linkage and transfer of knowledge in Latinamerican Public Universities. The general objetive is to present an alternative strategy based on comunity engagement in Higher education. The final conclution is to promote Public Higher Education in Latinamerica as an engine that produced equitable development in local contexts.
\end{abstract}

Keywords: Higher Education; Social Responsibility; Education Policy; Linking and Knowledge Transference.

\title{
Introducción
}

$\mathrm{L}$ a dinámica global del intercambio y la generación de conocimiento ha sido influenciada por los cambios tecnológicos, las formas de producción y las diferentes maneras de entender el mundo de acuerdo al contexto social. La experiencia en la investigación científica nos ha dejado una enseñanza: el mundo como lo entendemos hoy, es subjetivo; depende del avance de la tecnología y de los conocimientos profundos alcanzados por nuestros antecesores. Las explicaciones del mundo moderno dependen de nuestro conocimiento y también de nuestros contextos particulares, por ejemplo, hoy los científicos discuten sobre darle la categoría de planeta a Plutón; también la ciencia moderna ha demostrado que son más de tres estados de la materia, y esos conocimientos renovados se han incorporado a los planes y programas de estudio para hacer que el entendimiento de nuestro entorno sea homogéneo. La era de la tecnología y las comunicaciones nos obliga a estar abiertos a la posibilidad de que el conocimiento que hoy tenemos y los procesos en los que interviene el ser humano cambien en segundos. Como respuesta a estos escenarios, los países innovadores no sólo han desarrollado actividades en Investigación y Desarrollo ( $\left.\mathrm{I}^{+} \mathrm{D}\right)$, sino que han creado sus propios recursos humanos altamente calificados integrándolos satisfactoriamente al mundo del trabajo donde se transmite el conocimiento a través de actividades productivas.

El proceso de la vinculación inicia al identificar cómo se asimila y se transfiere el uso de los conocimientos generados en las universidades y los centros de investigación, complementándose con los conocimientos y experiencias de los trabajadores del sector productivo a través de los cuales se generan nuevas prácticas orientadas a la mejora productiva y por ende al desarrollo de la sociedad, de tal manera que el aprendizaje tecnológico y la transferencia 
de tecnología sea un proceso social continuo e interactivo y funcione como un sistema abierto dado que intervienen diversos actores. En primer lugar se encuentran los científicos que desarrollan el conocimiento, enseguida el sector productivo donde se aplican esos conocimientos, el sector social que se beneficia de ellos y el sector gubernamental que facilita estos intercambios. Por ello, la educación y las instituciones educativas son el semillero de personas capacitadas e ideas innovadoras que han transformado nuestro mundo.

En el contexto del neoliberalismo se han generado nuevas reglas del juego para el sector educativo. Las universidades y los centros de investigación a través de la investigación científica tienen la obligación de generar profesionistas que dominen conocimientos específicos para lograr resolver las necesidades sociales, como la pobreza, la vulnerabilidad y la escasez, Ahora más que nunca se requieren individuos preparados que desde su área del conocimiento promuevan la responsabilidad social, generen animación sociocultural y sean un factor de cohesión social para la búsqueda del bienestar colectivo. Por ello, la política educativa en México se ha centrado en ampliar la cobertura en educacion superior, con el fin de lograr llegar a más ciudadanos.

En México el 54.7\% del gasto federal en educación se destina al nivel básico, el $11 \%$ al medio superior y el $22.5 \%$ al superior; esta proporción incluye el gasto destinado a Ciencia y Tecnología (Segundo Informe de Gobierno: México, 2013). La proporción del presupuesto del nivel superior atiende a 253531 escuelas, 1842896 docentes y una población estudiantil de 34821326 alumnos (Anuario Estadístico. SEP, 2014), lo que arroja una relación de 18 estudiantes por cada docente contratado.

El total de la población mexicana entre 18 y 24 años, correspondientes a la edad promedio para atender la educación superior, asciende a 19073650 personas, de las cuales 450284 son analfabetas. La población total de 18 y más años, según datos del INEGI (2010), asciende a 71712388 personas, de las cuales el $81 \%$ no cuentan con instrucción superior, el $2 \%$ cuenta con estudios técnicos o comerciales con preparatoria terminada, el 15\% cuenta con algún grado de los estudios de licenciatura (en proceso o trunca) y sólo el 1.25\% con estudios de posgrado.

La formación de profesionistas especializados en diversas areas del conocmiento permite mejorar la competitividad de los países y ser un vínculo con la estructura de producción, promoviendo innovaciones constantes. Por ello, los países invierten cada vez más en educación y generación del conocimiento, pues esta inversión se refleja directamente en la producción nacional. Una muestra de ello se puede apreciar en la tabla 1, la cual enlista las regalías mundiales generadas por el uso de propiedad intelectual. 
Tabla 1

Regalías por el uso de propiedad intelectual en el mundo

\begin{tabular}{ccccc}
\hline Año & $\begin{array}{c}\text { América Latina y el } \\
\text { Caribe }\end{array}$ & Asia & Europa & Norteamérica \\
\hline 2005 & 530 & 21002 & 39084 & 77321 \\
\hline 2006 & 653 & 23288 & 40777 & 86909 \\
\hline 2007 & 915 & 26652 & 47728 & 101637 \\
\hline 2008 & 1081 & 30292 & 53964 & 106256 \\
\hline 2009 & 1023 & 27195 & 56821 & 102007 \\
\hline 2010 & 1048 & 32520 & 61532 & 110336 \\
\hline 2011 & 1342 & 36938 & 70777 & 126681 \\
\hline 2012 & 1039 & 40130 & 65980 & 129237 \\
\hline 2013 & 1096 & 39567 & 70469 & 132933 \\
\hline
\end{tabular}

Fuente: Banco Mundial (marzo 2015). Las cifras se expresan en millones de dólares.

Como se puede observar, en América Latina y el Caribe tan sólo se produce el $0.45 \%$ del total de regalías en el mundo, mientras que en Norteamerica se concentra el 54.47\%. De acuerdo con las cifras del Banco Mundial, entre los años 2015 y 2013, las regalías por el uso de propiedad intelectual en América Latina y el Caribe se duplicaron, mientras que en ese mismo periodo en Norteamerica, Europa y Asia sólo aumentaron entre 70\% y 80\%. Un tema que debe reflexionarse, es que el registro de propiedad intelectual no garantiza que la producción científica tenga un beneficio colectivo, tal es el caso del crecimiento economico desmesurado en manos de unos pocos habitantes del mundo.

En América Latina la investigación científica se realiza sobre todo en las Universidades Públicas, pero la globalización ha generado nuevas reglas del juego. La era de las comunicaciones y la tecnología ha dado pie a la expansión del conocimiento a un ritmo vertiginoso. La competitividad en todos los ámbitos cobra mayor relevancia y resulta trascendental que las universidades se pongan en contacto con las necesidades detectadas en su región. Este vínculo entre las universidades y la sociedad se produce de diversas maneras, primero, como respuesta a las demandas de capacitación del sector productivo, donde las universidades ofertan carreras y capacitación especializada a través de la formación continua, y segundo, a través de la investigación y el desarrollo con aplicación práctica, ya sea por medio de proyectos de investigación financiados por gobierno o a través de la consultoría.

Esto pone en evidencia la importancia de estudiar el concepto de vinculación como un eje fundamental de la vida académica que permitirá contribuir al desarrollo entendido como "[...] la mejora constante del nivel de vida de 
los integrantes de una sociedad de forma que éstos puedan no sólo satisfacer sus necesidades básicas, sino aquellas que le son importantes según sus prioridades" (Castells, 2007). Aunque actualmente es poco probable establecer una única definición del concepto de vinculación, destaca para este documento la prevalencia de las relaciones existentes entre la academia, el conocimiento que ahí se produce y la manera en como transforma su contexto local. En el siguiente apartado se revisan con mayor profundidad los conceptos de vinculación y transferencia del conocimiento.

\section{Vinculación y Transferencia del conocimiento: Condiciones y realidades}

El término vinculación está ligado totalmente a la gestión del conocimiento en los centros de investigación y universidades en el mundo, y es a través de él donde se genera la transferencia de conocimientos que produce resutados positivos en la sociedad, ya sea con nuevas tecnologías, innovación en productos o modificaciones a los procesos productivos. Al ser adoptado por el sector productivo, se entiende que el conocimiento será utilizado para generar desarrollo social y mejorará la calidad de vida de los habitantes de la región que tenga acceso a ese nuevo conocimiento.

Desde el punto de vista universitario, la vinculación es el proceso integral que articula las funciones sustantivas de docencia, investigación, extension de la cultura y los servicios de las instituciones públicas de educación superior (IES) para su interacción eficaz y eficiente con el entorno socio-económico mediante el desarrollo de acciones y proyectos de beneficio mutuo, que contribuyan a su posicionamiento y su reconocimiento social (Alvarado, 2009).

Para las Instituciones Públicas de Educación Superior en México, la vinculación con el sector productivo es parte fundamental de la pertinencia que ellas mismas reconocen en sus principios rectores. Con la vinculación, los centros educativos consolidan su pertinencia social a través de la formación de profesionales que puedan producir ideas, procesos, productos o servicios creativos e innovadores que resulten en el bienestar y el desarrollo de la sociedad en su conjunto, pues su formación universitaria les permite ser agentes de cambio a través de los conocimientos, aptitudes y actitudes que se imparten en las instituciones de educación superior.

El ejercicio de la vinculación suele formalizarse mediante convenios de colaboración legalmente establecidos con los sectores productivo y gubernamental. Éste ha sido el primer paso para que las Instituciones de Educación Superior accedan a recursos financieros alternos que le permitan fortalecer 
su infraestructura de investigación: aulas, laboratorios y talleres, entre otros espacios formativos, y propicien estímulos y reconocimientos a los investigadores que participan en el proceso de generación de ciencia y tecnología.

En los últimos años se ha puesto especial énfasis en el impulso a la relación Educación-empresa-asociaciones civiles y gobierno, pues los grandes problemas del mundo requieren la participación de todos, de manera individual y grupal. Por ello la cooperación con asociaciones civiles es un tema que las Universidades en América Latina deberán explorar en los próximos años. Otros retos relacionados con la vinculación y la transferencia del conocimiento están relacionados con:

- El enfoque de la investigación: básica o aplicada orientada a la solución de problemáticas mundiales, nacionales, regionales y locales.

- La construcción de ciudadanía, capital social y cohesión social para producir el crecimiento económico y propiciar el desarrollo de los países.

- La calidad educativa debe hacerse comparable y transferible incluso en los sistemas públicos y privados. Esta acción es un reto que la evaluación educativa no ha logrado resolver.

- Las Instituciones de Educación Superior deben ser los promotores de la responsabilidad social y la sustentabilidad.

- El uso de la tecnología en los procesos educativos debe incrementarse sobretodo en América Latina, donde la conectividad y la infraestructura aún no son una realidad.

Para contextualizar el concepto de vinculación, debemos conocer los antecedentes históricos, pues la brecha tecnológica ha generado una dependencia total por parte de los países subdesarrollados hacia los industrializados. Históricamente esta dependencia fue hacia los productos manufacturados y en la actualidad está enfocada en la tecnología de punta.

\section{Evolución de la vinculación y la trasferencia del conocimiento en América Latina: una propuesta basada en la Responsabilidad Social Universitaria}

En América Latina existe un atraso significativo con respecto al desarrollo de la política científica, pues esta región se ha caracterizado principalmente por ser productora de materia prima y recursos humanos de poca calificación. Los gobiernos latinoamericanos promueven políticas económicas que evidencian este escenario como una oportunidad para las empresas transnacionales, sobre todo por el bajo costo de la mano de obra y por el acceso a los 
recursos naturales de esta región. Los países de América Latina destinan en promedio el $27.38 \%$ de su producción a la industria, $8.55 \%$ a la agricultura y el $64.07 \%$ a la prestación de servicios (CIA, 2012). Llama la atención que la producción de paises como Panamá y Cuba se concentra en más del 80\% en la prestación de servicios, seguido por Costa Rica, Uruguay, Belice y Brasil donde los servicios representan entre el 70\% y 74\% de la producción. Es un hecho que los paises latinoamericanos deben buscar una opción de desarrollo a través de la producción agricola.

Por estas razones, el gobierno, las universidades, las asociaciones civiles y las industrias de Latinoamerica deben buscar la manera de detonar sistemas de innovación que permitan revertir las condiciones socioeconómicas de la región. Para lograrlo, estos cuatro sectores deben tener un vínculo estrecho y en consecuencia:

1. El sector educativo debe ofertar carreras acordes a los mercados laborales.

2. El sector productivo debe aprovechar el conocimiento generado en las instituciones educativas.

3. El sector social debe trabajar de la mano con universidades y centros de investigación con el fin de difundir y apropiarse de los resultados sistémicos de la producción científica en todas las áreas del conocimiento.

4. El gobierno debe generar políticas públicas que favorezcan el intercambio y la aplicación de nuevas tecnologías en beneficio de la sociedad.

Por su parte, el neoliberalismo, la globalización y la competencia voraz han obligado a que los actores e instituciones busquen nuevas formas de organización para lograr que la transferencia del conocimiento sea real y tenga impacto directo en las economías nacionales. El proceso histórico que ha marcado la evolución de la vinculación y la transferencia del conocimiento no ha sido fácil.

Históricamente el estado benefactor en América Latina concentró todos los esfuerzos de vinculación en la burocracia. Las instituciones de educación superior turnaban sus investigaciones a la administración central, la cual enviaba el reporte de los avances tecnológicos directamente al gobierno, donde finalmente se administraban los nuevos conocimientos para ser aplicados en las empresas paraestatales. Esta tendencia aun está presente en muchas decisiones académicas, tanto por su arraigo histórico como por la centralización del poder estatal, a pesar de que en el contexto moderno se ha comprobado la eficacia de la descentralización. Por tanto, la educación superior en América Latina se ve atada por los procesos históricos que han marcado las pautas de comportamiento de la política educativa en ciencia y tecnología. 
Como consecuencia a esta tendencia, surge la escuela del pensamiento latinoamericano en Ciencia y Tecnología del Desarrollo, impulsada por Jorge Sabato y Natalio Botana (1968) con la idea de que el conocimiento generado en las universidades debía transferirse directamente al sector productivo y que el Estado era quien debía fomentar esta vinculación. Los primeros países que dieron apertura a esta idea fueron Argentina y Brasil que generaron los primeros intentos de vinculación al implementar un modelo que buscó la transferencia de tecnología directa, sin pasar por el estado. Esta corriente de pensamiento no tuvo eco en el resto de América Latina, sin embargo fue en Brasil donde los resultados no fueron despreciables.

La revisión bibliográfica muestra que actualmente se identifican nuevas tendencias teóricas que ponen de manifiesto el papel del conocimiento como motor de cambio en una sociedad. Esta riqueza no depende del desarrollo tecnológico en sí mismo, sino de la evolución del conocimiento y el consenso que se genera en el imaginario social, y de esta forma experimenta nuevas maneras de entender el mundo. Este conocimiento produce trabajadores y profesionales calificados, que desarrollan las tecnologías, ingeniería gerencial y descubrimientos científicos para ser utilizados en la creación de nuevos productos para el beneficio de la sociedad, siendo éste el fin último del conocimiento.

Las ideas y estrategias utilizadas en el desarrollo de la vinculación y la transferencia del conocimiento nos permiten proponer un esquema de análisis para América Latina centrado en cuatro etapas que obedecen a momentos históricos representativos:

1. Vinculación centralizada.

2. Vinculación descentralizada.

3. Tecno-vinculación.

4. Vinculación con responsabilidad social territorial.

Esta propuesta de análisis busca abonar al desarrollo de la política educativa, presentando una síntesis de información producto de la revisión bibliográfica. El modelo incorpora el estudio de 5 dimensiones para cada etapa histórica (ver tabla 4):

a) Contexto educativo en América Latina.

b) Crecimiento del número de Instituciones de Educación Superior en América Latina.

c) Características generales de la vinculación en cada etapa histórica.

d) Mecanismos para la transferencia del conocimiento.

e) Organización interna para la vinculación y la transferencia del conocimiento. 
Tras esta propuesta, basada en el análisis histórico y complementada con los procesos actuales, la reflexión obligada para los líderes educativos en América Latina debe ser replantear la misión de la educación superior, pues el modelo educativo basado en competencias - propuesto por el proceso Bolonia (2010) que estableció el Espacio Europeo de Educación Superior - está generando profesionales súper-especializados con un curriculum académico que pierde vigencia año con año (De Garay, 2012). En América Latina este sistema educativo ha producido "profesionistas obreros", que no pueden competir por un salario equivalente al ofertado en países industrializados debido a la alta demanda de empleos y a la débil oferta laboral.

Desde esta perspectiva, la "responsabilidad social" se presenta como una opción para que las Instituciones Públicas de Educación Superior incidan de manera efectiva en el bienestar colectivo, la inclusión, la sostenibilidad y la superación de la vulnerabilidad; esta última es una constante en los países integrantes de esta región.

Definimos a la Responsabilidad social como la finalidad del bienestar colectivo, pues a través de las redes colaborativas que se gestan en las aulas universitarias se pueden construir valores como la responsabilidad y se adquieren conocimientos que nos permiten tomar decisiones fundamentadas en beneficio propio y de los demás. Por lo tanto estas instituciones deben reflexionar acerca de los resultados positivos del conocimiento, los valores y comportamientos que promueven en sus aulas, pues la universidad es un sistema de producción de saberes donde confluyen los individuos que más adelante orientarán el rumbo social en terminos económicos y políticos.

Considero que una estrategia efectiva para practicar la responsabilidad social desde las Instituciones de Educación Superior puede aplicarse desde tres ópticas:

a) Responsabilidad social interna: los valores que comparte un grupo de invididuos en particular por el hecho de pertenecer a una institución. Hoy las instituciones educativas han dejado de formar en valores y sólo atienden los procesos técnicos que el mercado global exige. La competencia es el centro de la toma de desiciones.

b) Responsabilidad social externa: los espacios que provee la Universidad para ser sede de reuniones con miembros de la comunidad con un fin en particular: fortalecer la cohesión social. Estos espacios pueden generarse para difundir la cultura, el conocimiento y la investigación aplicada; los espacios de emprendedurismo son un buen ejemplo, también la colaboración directa entre Asociaciones Civiles y miembros de la comunidad Universitaria. Estas redes fortalecen el tramado social y permiten que la investigación que se lleva a cabo en las Instituciones Educativas de nivel Superior pueda ser aplicable y beneficie de manera directa a los miembros 
de la comunidad, pues constantemente permite el intercambio de opiniones y la deliberación pública de la ciencia.

c) Responsabilidad social indirecta: obedece a los valores cívicos que se deben reproducir como sello personal de una formación universitaria; impactan de manera indirecta en una comunidad, pues se invierte en el presente, para que en el futuro cercano sus egresados sean individuos proactivos en la búsqueda del bienestar colectivo y la supervivencia humana. En este nivel, las Instituciones de Educación Superior inciden en las relaciones con el Estado, la sociedad, el desarrollo y el medio ambiente global a traves de sus egresados.

El gran reto es construir ciudadanía desde las universidades, como una manera de evitar daños futuros y generar condiciones para el desarrollo de la cohesión social, pues la diversidad humana permite que todos y cada uno de los individuos de una comunidad desarrollen diversas capacidades; pueden ser formales, como las que se generan a través de la educación escolar, pero también informales, como las experiencias de vida. Estas capacidades nos hacen diferentes a los demás individuos, incluso cuando somos producto de un mismo núcleo social.

El punto no está en contabilizar nuestros conocimientos o experiencias, sino en tratar de que estas fortalezas puedan incidir de manera benéfica en nuestro entorno. En otras palabras, es deber de las Instituciones de Educación Superior promover la investigación aplicada que favorezca el bienestar colectivo, la justicia y la equidad mediante procesos de vinculación y transferencia del conocimiento dirigidos a resolver problemas reales en condiciones reales.

\section{Conclusiones}

Tras el análisis se destaca el rol que tienen las Instituciones Públicas de Educación Superior como factor de cambio en la construcción de una ciudadanía activa y responsable, pues es en las aulas donde se reproducen los valores cívicos y morales que permiten el desarrollo económico y social de las comunidades. Este deber social también se debe reproducir en la vinculación y la transferencia del conocimiento hacia los estudiantes y la comunidad en la cual los investigadores pueden incidir de manera directa.

Las instituciones educativas han tenido que preocuparse por responder a las demandas de la industria o del sector productivo, y como resultado de esto se ha generado educación superior sin valores ni ideologia que permita fortalecer alguna identidad colectiva, pues sólo educamos para responder a las demandas del mercado, sin incorporar las necesidades sociales de nues- 
tro contexto. Por tanto, la educación superior se debe reorganizar incorporando en sus decisiones el impacto que éstas puedan tener en los grupos sociales que la integran.

De esta manera se espera producir un escenario que fortalezca la cohesión social entre los miembros de una comunidad, pues cuando las Instituciones de Educación Superior públicas o privadas generan conocimiento, lo hacen con el único fin de favorecer a la sociedad. La vinculación y la transferencia del conocimiento entre las universidades y la sociedad deben ser mas estrechas, pues la responsabilidad social es un compromiso moral irrenunciable que poseen las instituciones educativas para aplicar el conocimiento que se produce a traves de la investigación, con una visión más humanista. El papel prioritario de las universidades debe ser instituir la Responsabilidad Social de la Ciencia gracias a una gestión social del conocimiento que beneficie a todos los actores sociales.

\section{Referencias}

Alvarado Borrego A. (2009). Vinculación universidad-empresa y su contribución al desarrollo regional. Ra Ximhai Revista de Sociedad, Cultura y Desarrollo Sustentable. Septiembre-diciembre, año/Vol. 5, Número 3. pp. 407-414. México: Universidad Autónoma Indígena de México. ISSN: 1665-0441

Banco Mundial (2015). Cargos por el uso de propiedad intelectual, pagos. Consultado en: http:// datos.bancomundial.org/indicador/BM.GSR.ROYL.CD

Castells, D. (2007). Otro concepto de Desarrollo. Constribuciones a la Economía. IssN: 16968360 eumed. Consultado en http:/ / www.eumed.net/ce/2007b/dc-0711.htm

CIA (2012). The World Factbook Central Intelligence Agency. Consultado en: https:// www.cia.gov/library/publications/the-world-factbook/fields/2012.html

Coneval (2015). Pobreza en México. Consultado en: http://www.coneval.gob.mx/ Medicion/MP/Paginas/Pobreza_2014.aspx

De Garay A. (2012). Los 10 primeros años del proceso de Bolonia en la Educación Superior en Europa. Revista de la Educación Superior. Vol. XLI (2), No. 162. AbrilJunio de 2012, pp. 113-126

INEGI (2010). Censo Nacional de Población y Vivienda. México: INEGI.

INEGI (2014). Cobertura en Educación Superior. Anuario Estadístico y Geográfico por Entidad Federativa. México: INEGr. Consultado en: http://www.inegi.org.mx/ prod_serv/contenidos/espanol/bvinegi/productos/integracion/pais/aepef/2014/702825063986.pdf

Presidencia de la República Mexicana (2013). Segundo Informe de Gobierno del Presidente Enrique Peña Nieto. Consultado en: http://www.presidencia.gob.mx/segundoinforme/

RAE (2015). Vinculación. Diccionario de la Real Academia Española. Consultado en: www.rae.es 
SEP (2014). Anuario Estadístico de Instituciones de Educación Superior en México. Secretaría de Educación Pública. Consultado en: www.sep.gob.mx En julio de 2015.

UNESCO (2009). Instituto de Estadística. Consultado en: http:/ / www.unesco.org/new/ es/education/themes/planning-and-managing-education/policy-and-planning/capacity-development/

Valenti G. (2011). Construyendo puentes entre el capital humano y el sistema de innovación. México: Flacso. 